\title{
CESAREA EXTRAPERITONEAL
}

\section{Estudio Comparativo}

\author{
Dr. Jorge Milanés Pernett* \\ Dr. Alberto Zabaleta Lombana** \\ Dr. Antonio Soto Yances***
}

Universidad de Cartagena - Facultad de Medicina - Dpto. de Obstetricia y Ginecología

\section{INTRODUCCION}

En ocasión pasada presentamos a la consideración de Uds. en el $V$ Congreso reunido en Medellín, un opúsculo sobre Cesárea Extraperitoneal con el fin de llamar la atención hacia una técnica quirúrgica ya casi en el olvido debido a sus múltiples inconvenientes y desagradables complicaciones, mas parece que el golpe de gracia le fue dado por el advenimiento de los antibióticos. Pues bien, con la técnica seguida por nosotros, ya expuesta en el trabajo anterior, logramos obviar unos y reducir al mínimum otros. Esto nos animó a seguirla practicando con más regularidad y a usarla, no solamente en los casos con indicación precisa, sino también en aquellos en los cuales se podía usar sin inconvenientes la transperitoneal. Comoquiera, que en el porvenir tanto inmediato, como mediato y tardio hemos observado algunos resultados tan evidentes que nosotros

\footnotetext{
* Jefe del Depto. de Obstetricia y Ginecología.

** Profesor Asistente de Obstetricia y Ginecología.

*** Residente II de Obstetricia y Ginecología.
}

consideramos altamente satisfactorios, nos ha llevado a establecer algunos puntos de comparación con la cesárea transperitoneal, tan difundida y practicada con éxito innegable hoy en día.

La casuística presentada por nosotros es tan reducida en comparación con la casuística de la cesárea transperitoneal, tan fácil y sencilla, aún en manos poco expertas, que muchas de las ventajas consideradas podrían tornarse en desventajas en igualdad de condiciones, tanto de paciente como de habilidad del cirujano.

\section{MATERIAL Y METODOS}

Como material para el presente trabajo se tomaron todas las cesáreas practicadas en la Clínica Universitaria de Maternidad "Rafael Calvo C." de Cartagena, durante el lapso comprendido entre el 1\% de Enero de 1964 y el 10 de Octubre de 1965.

En la práctica de la Cesárea Transperitoneal intervino todo el Personal Docente y Residentes de la Clínica y en la extraperitoneal solo los autores del presente trabajo. 
Los resultados obtenidos son los siguientes:

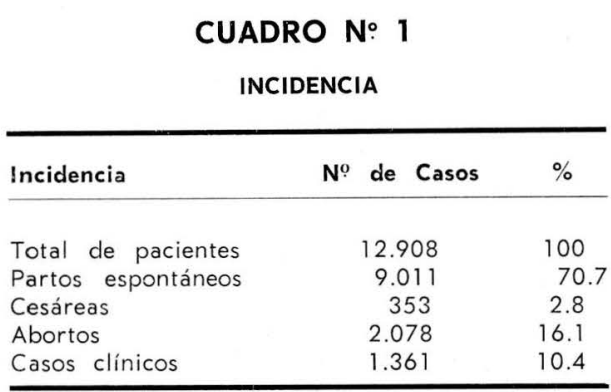

El cuadro anterior presenta un total de 12.908 pacientes los cuales ingresaron a la Clínica en el lapso mencionado anteriormente y correspondiendo un número de 353 a quienes fue necesario practicar cesárea, lo cual representa el $2.8 \%$ del total.

\section{CUADRO N: 2}

TIPO DE CESAREA

\begin{tabular}{|c|c|c|c|c|}
\hline Tipo de C & Cesárea & $N^{0} \quad d$ & de Casos & $\%$ \\
\hline \multicolumn{5}{|c|}{ Cesárea segmentaria } \\
\hline Transp. & & & 315 & 90.2 \\
\hline Segmento & corporal Transp. & & 14 & 3.3 \\
\hline Extraperito & tonea! & & 23 & 6.5 \\
\hline Vaginal & & & 1 & - \\
\hline TOTAL & & & 353 & 100 \\
\hline
\end{tabular}

De los 353 casos de cesáreas, correspondieron 315 a cesáreas transperitoneal segmentaria o sea el $90.2 \%$ y 23 a extraperitoneal, lo cual significa el $6.5 \%$ de todas las cesáreas.

Entre los 23 casos presentados de extraperitoneal corresponden 3 en los cuales la Cesárea fue a repetición y uno de ellos por tercera vez. El caso de la cesárea vaginal se practicó por prolapso genital total con un embarazo a término.
CUADRO NN: 3

EDAD

\begin{tabular}{lcc}
\hline Grupo de Edades & $\mathbf{N}^{0}$ de Casos & $\%$ \\
\hline $14-19$ & 8 & 34.8 \\
$20-24$ & 6 & 26.1 \\
$25-29$ & 4 & 17.4 \\
40 & 1 & 4.3 \\
TOTAL & 23 & 100 \\
\hline
\end{tabular}

La edad de las pacientes fluctuó entre los 14 y los 43 años, siendo más frecuente entre los 14 y los 19 y entre los 20 y los 24 , encontrándose entre ellas dos pacientes con 14 años y una de 43.

CUADRO N: 4

GRAVIDEZ

\begin{tabular}{lcc}
\hline Gravidez & $N^{0}$ de Casos & $\%$ \\
\hline Primigestante & 12 & 52.2 \\
Bigestas & 4 & 17.4 \\
Trigest. & 3 & 13.0 \\
Multigest. & 4 & 17.4 \\
TOTAL & 23 & 100 \\
\hline
\end{tabular}

La gravidez detallada en el cuadro anterior revela que el mayor número de casos fue en primigestantes jóvenes y que en las multigestantes la intervención se practicó con menor intensidad. La mayor gravidez para una paciente fue de 10, seguida de una con ocho gestaciones.

Entre el grupo de pacientes, cuatro tenían antecedentes de cesárea transperitoneal y tres pacientes con antecedentes de cesárea extraperitoneal, una de ellas con dos anterior.

CUADRO N: 5

EDAD DE LA GESTACION

\begin{tabular}{|c|c|c|c|}
\hline Semana & $N^{o}$ & de Casos & $\%$ \\
\hline $34-36$ & & 7 & 30.8 \\
\hline $36-42$ & & 16 & 69.2 \\
\hline TOTAL & & 23 & 100 \\
\hline
\end{tabular}


La edad de la gestación varió entre las 34 y 42 semanas, encontrándose la mayor frecuencia entre las 36 y 42 semanas con un $69.2 \%$.

Solo un caso refirió edad gestacional de 42 semanas, probablemente por error cronológico.

\section{CUADRO N: 6}

DURACION PARA LA EXTRACCION DEL FETO

\begin{tabular}{lcc}
\hline Minutos & $N^{\mathbf{0}}$ de Casos & $\%$ \\
\hline 4 & 2 & 8 \\
5 & 7 & 30.5 \\
6 & 2 & 8.6 \\
7 & 3 & 13.1 \\
8 & 2 & 8.6 \\
10 & 3 & 13.1 \\
12 & 4 & 17.5 \\
TOTAL & 23 & 100 \\
\hline
\end{tabular}

Hemos considerado de gran importancia el tiempo empleado para la extracción del feto, sobre todo en aquellos casos de sufrimiento fetal, obteniendo el cuadro anterior en que nos muestra que el tiempo empleado por nosotros fluctuó entre los 4 y 12 minutos, teniendo siete casos en los cuales empleamos cinco minutos $y$ dos con duración de cuatro minutos. El tiempo máximo de 12 minutos correspondió a la paciente con dos cesáreas extraperitoneales anteriores y otras con problemas de adherencia por cirugía anterior.

\section{CUADRO $\mathrm{N}: 7$} CAUSAS

\begin{tabular}{lcc}
\hline Entidad & $N^{\mathbf{9}}$ de Casos & $\%$ \\
\hline Amnionitis & 18 & 78.2 \\
Distocia feto Mat. & 11 & 47.1 \\
T. de P. P. & 8 & 34.2 \\
Suf. fetal & 4 & 17.1 \\
Pre-Rupt. Ut. & 3 & 13.1 \\
Dist. de Cont. & 2 & 8.7 \\
Bartholinitis Sup. & 1 & 4.2 \\
T.B.C. Pulm. & 1 & 4.2 \\
Dep. Prem. Plac. & 1 & 4.2 \\
Hematoma vulvar & 1 & 4.2 \\
Primigesta añosa & 1 & 4.2 \\
\hline
\end{tabular}

Por lo general el diagnóstico fundamental sobre el cual nos basamos para practicar las Cesáreas extraperitoneales, fue la amnionitis, no obstante haberla practicado en casos no infectados y aún en aquellos en los cuales se podría utilizar sin inconvenientes la transperitoneal.

En el cuadro anterior se enumeran las causas tomadas con justificación para la cesárea extraperitoneal.

\section{TECNICA QUIRURGICA}

En la mayoría de las cesáreas transperitoneales se usó como material de sutura el cat-gut. Sin embargo en todos los casos de cesáreas extraperitoneales se usó seda 80 en todas las suturas, desde músculo uterino hasta la piel y como apósito se usó una pequeña gasa empapada en alcohol la cual fue removida a las 24 horas, habiéndose retirado los puntos a las 48 horas y levantado a la paciente a las 96 horas.

\section{CUADRO № 8}

\section{PESO DEL FETO}

\begin{tabular}{lcc}
\hline Peso & N $^{0}$ de Casos & $\%$ \\
\hline $2.500-2.999$ & 3 & 12.2 \\
$3.000-3.499$ & 8 & 34.2 \\
$3.500-3.999$ & 8 & 34.2 \\
4.000 o más & 4 & 18.4 \\
TOTAL & 23 & 100 \\
\hline
\end{tabular}

Los pesos de los niños se resumen en el cuadro anterior, observándose que la mayoría fluctuó entre los 3.000 y los 4.000 gramos.

\section{ESTADO DEL FETO}

De los 23 fetos, 17 fueron perfectamente normales y no tuvieron complicaciones inmediatas, cinco estaban apneicos y fue necesario llevarlos a incubadoras y administrarles oxígeno para su recuperación. Solo un niño 
nació muerto y correspondió al caso de desprendimiento prematuro de placenta.

El estado del feto se resume en el cuadro que sigue.

CUADRO $\mathrm{N}: 9$

ESTADO DEL FETO

\begin{tabular}{|c|c|c|c|}
\hline Estado del feṙo & $N^{0} d e$ & Casos & $\%$ \\
\hline Bien & 1 & & 73.9 \\
\hline Apneico & & 5 & 21.7 \\
\hline Muerto & & 1 & 4.4 \\
\hline
\end{tabular}

\section{CUADRO NN 10}

\section{MORBILIDAD \\ EVOLUCION - COMPLICACIONES}

\begin{tabular}{lrrrrr}
\hline & \multicolumn{2}{c}{$\begin{array}{c}\text { Transperi- } \\
\text { toneal }\end{array}$} & \multicolumn{3}{c}{$\begin{array}{c}\text { Extraperi- } \\
\text { toneal }\end{array}$} \\
Complicación & $\begin{array}{l}\mathbf{N}^{\mathbf{0}} \text { de } \\
\text { Casos }\end{array}$ & $\begin{array}{l}\text { No de } \\
\text { Casos }\end{array}$ & $\%$ \\
\hline Inf. herida & 62 & 18.7 & 1 & 4.5 \\
Dehiscencia sutura & 6 & 1.9 & - & - \\
Endometritis & 10 & 3.3 & - & - \\
Fiebre & 60 & 18.2 & 4 & 17.4 \\
Evisceración & 1 & 0.3 & - & - \\
Otras & 8 & 2.4 & 2 & 8.8 \\
Ninguna & 163 & 55.2 & 16 & 69.3 \\
TOTALES & 330 & 100 & 23 & - \\
\hline
\end{tabular}

A la dificultad de la técnica se sumaban las complicaciones post-operatorias de todo orden, ocupando sitio muy destacado las supuraciones de la incisión quirúrgica que llegaban en algunos casos a la mortificación y esfacele de sectores considerables de tejidos, dejando cicatrices indeseables y prolongado por un tiempo desconcertante la invalidez de la paciente. Hoy en día con el mejoramiento de las técnicas y el uso de los antibióticos, todo ese cuadro aterrador ha cambiado radicalmente, según lo exponemos en el cuadro anterior (Cuadro № 10), en clonde se observa que la complicación más frecuente fue la fiebre inespecífica, la cual atribuímos nosotros a procesos infecciosos diferentes a la herida quirúrgica, tales como pielonefritis, mastitis, etc.

\section{RESUMEN}

Se presentan 23 casos de Cesárea extraperitoneal, practicadas en la Clínica Universitaria de Maternidad "Rafael Calvo C." de Cartagena, en el lapso comprendido entre el $1 \%$ de Enero de 1964 y el 10 de Octubre de 1965, las cuales representan el $6.5 \%$ de todas las cesáreas afectadas en dicho centro asistencial.

Se hace notorio que la mayoría de estos casos se presentaron en pacientes primigestantes, entre los 14 y los 19 años y que el $78.2 \%$ de los casos fueron impuros, es decir con infección manifiesta, generalmente amnionitis.

Igualmente se hace un esquema ilustrativo de la incidencia de la operación cesárea, relacionándola con el número total de pacientes ingresados a la Clínica, así como el tipo de cesárea practicada en cacla caso.

Se detalla la edad de las pacientes y la gravidez de las mismas, señalándose además, la edad de la gestación y la duración para la extracción del feto, partiendo del momento de la anestesia, punto que consideramos de extrema importancia, especialmente en los casos de sufrimiento fetal.

Se enumeran las causas fundamentales que justificaron la intervención y se habla brevemente de la conducta seguida por nosotros durante el post-operatorio.

Presentamos datos sobre el peso en gramos del feto obtenido en la operación y el estado del mismo, explicando el manejo inmediato y el tratamiento para la recuperación pronta de los apneicos. 
Finalmente hacemos un cuadro comparativo entre la cesárea transperitoneal y la extraperitoneal, teniendo en cuenta que, durante el post-operatorio se tuvieron los mismos cuidados y tratamientos en forma rutinaria, encontrándose que el $18.7 \%$ de los casos y transperitoneal (62) casos sufrieron infección de la herida y solo 1 caso de las extraperitoneales se infectó, transcurriendo 16 casos, en los cuales no hubo ninguna complicación, no obstante que la mayoría de las pacientes eran casos impuros.

\section{CONCLUSIONES}

1․ Es muy recomendable que la cesárea extraperitoneal forme parte del arsenal terapéutico del obstetraginecólogo.

2: La práctica de ésta no presenta hoy en día los inconvenientes de otra época.

3: La asepsia y antisepsia, manejo cuidadoso de los planes y el uso de los antibióticos, nos ayudan a obtener un post-operatorio libre de complicaciones.

4. Con la técnica y cuidados empleados por nosotros, cortamos los puntos a las 48 horas y levantamos la paciente a las 96.

5․ Aún en los casos en que la practicamos por sufrimiento fetal, fue ejecutada con éxito y en un tiempo que permitió presentar auxilios de reanimación del feto.

6: La morbilidad por infección de la incisión operatoria fue mucho me- nor en la extraperitoneal, que en la transperitoneal.

7․ La cesárea extraperitoneal a repetición no presentó inconvenientes.

8: En la práctica de esta intervención no hubo accidentes vesicales, ni uretrales y el post-operatorio los problemas urinarios no fueron mayores que en la práctica de las otras cesáreas.

\section{BIBLIOGRAFIA}

1 ELLIS G. J., DEVITA M. R.: Extraperitoneal cesarean section. Am. J. Obst. Gynec. Vol. 82. No 3 Septiembre de 1961. Pág. 695.

2 Bourgeols G. A., PHANEEUF L. E.: Am. J. Obst. Gynec. La vía parasupravesical bilateral en la operación cesárea extraperitoneal. Reproducido en América Clínica. Octubre de 1952.

3 Actas Ciba. La operación cesárea. JulioSeptiembre 1952.

4 PEREZ MANUEL. Tratado de Obstetricia. Sexta Edición. Págs. 932-948.

5 BOtella Llusia J. Ginec. Pract. Vol. 8. Pág. 85. 1957.

6 GREENHILL J. P. Principios y Práctica de Obstetricia De Delee. II Edición. Edit. Uthea. Págs. 1.242- 1955.

7 RAMIREZ MERCHAN R. F.: A propósito de cesárea extraperitoneal. Rev. Médica. Vol. 49. 564-691. 1946.

8 WATERS E. G. Supravesical Extraperitoneal cesarean section. Clinical Obst. and Gynec. Pág. 985. 1959.

9 WATERS E. G. Extraperitoneal cesarean section. Am. J. Obst. and Gynec. Vol. 49 7391945.

10 MILANES J. ZABALETA A. Cesárea extraperitoneal. Rev. Col. Obst.-Ginec.

11 MILANES J. ZABALETA A. Cesárea extraperitoneal. Algunas consideraciones. Rev. Col. Obst. y Ginec. Vol. XV. Nọ 4 Pág. 341. Jul.-Agosto 1964. 